\title{
Shatter: Efficient Symmetry-Breaking for Boolean Satisfiability
}

\author{
Fadi A. Aloul, Igor L. Markov, Karem A. Sakallah \\ Department of EECS, University of Michigan \\ \{faloul, imarkov, karem\}@eecs.umich.edu
}

\begin{abstract}
Boolean satisfiability (SAT) solvers have experienced dramatic improvements in their performance and scalability over the last several years $[5,7]$ and are now routinely used in diverse EDA applications. Nevertheless, a number of practical SAT instances remain difficult to solve [9] and continue to defy even the best available SAT solvers $[5,7]$. Recent work pointed out that symmetries in the Boolean search space are often to blame. A theoretical framework for detecting and breaking such symmetries was introduced in [2]. This framework was subsequently extended, refined, and empirically shown to yield significant speed-ups for a large number of benchmark classes in [1].

Symmetries in the search space are broken by adding appropriate symmetry-breaking predicates (SBPs) to a SAT instance in conjunctive normal form (CNF). The SBPs prune the search space by acting as a filter that confines the search to non-symmetric regions of the space without affecting the satisfiability of the CNF formula. For symmetry breaking to be effective in practice, the computational overhead of generating and manipulating the SBPs must be significantly less than the run time savings they yield due to search space pruning. In this paper we present several new constructions of SBPs that improve on previous work. Specifically, we give a linearsized CNF formula that selects lex-leaders (among others) for single permutations. We also show how that formula can be simplified by taking advantage of the sparsity of permutations. We test these improvements against earlier constructions and show that they yield smaller SBPs and lead to run time reductions on many benchmarks.
\end{abstract}

\section{Categories and Subject Descriptors}

T0.1 Fundamental CAD Algorithms

\section{General Terms}

Algorithms, Experimentation, Verification, Routing

\section{Keywords}

SAT, CNF, backtrack search, symmetries, clause learning, logic simplification, graph automorphism

\section{Introduction}

Many search, synthesis, and optimization problems arising in algorithmic applications exhibit symmetries. The presence of multiple, symmetric solutions may slow down known algorithms for such problems. Symmetries can make it more difficult to conclude that a a given instance of a search problem has no solutions because symmetric sub-instances may be independent. However, once the symmetries are identified, it is often easy to eliminate parts of the search space and, thereby, simplify the solution process.

Permission to make digital or hard copies of all or part of this work for personal or classroom use is granted without fee provided that copies are not made or distributed for profit or commercial advantage and that copies bear this notice and the full citation on the first page. To copy otherwise, or republish, to post on servers or to redistribute to lists, requires prior specific permission and/or a fee.

DAC 2003, June 2-6, 2003, Anaheim, California, USA.

Copyright 2003 ACM 1-58113-688-9/03/0006...\$5.00.
In this paper we study the Boolean satisfiability problem-one of the most important in Computer Science-in the presence of symmetry. Crawford et al. [2] laid the theoretical foundation for generating symmetry-breaking predicates for CNF formulas that possess permutational symmetries. The basic idea is to detect all such symmetries using a reduction to graph automorphism. For each such symmetry, an $\mathrm{SBP}$ - whose CNF representation is quadratic in the number of problem variables-is constructed. A SAT solver is then applied to the conjunction of the original formula and the SBPs created from each permutation in the group of symmetries. This approach breaks all permutational symmetries and will be referred to as full symmetry breaking. The downside is that the number of symmetries in the symmetry group is usually exponential in the number of problem variables making such full symmetry breaking impractical. They reduce the number of symmetries that need to be considered by building a symmetry tree and pruning it to remove unnecessary duplication. This, unfortunately, still does not preclude the need to consider an exponential number of permutations in order to break all symmetries.

This general framework was extended in [1] to handle phaseshift symmetries and their composition with permutational symmetries. Additionally, more efficient, albeit still quadratic, CNF constructions of the symmetry-breaking predicates were introduced. Finally, symmetry-breaking was only applied to the generators of the symmetry group as opposed to the entire set of symmetries. They argued, and presented strong empirical evidence, that full symmetry breaking is unnecessary and that such partial symmetry breaking is often quite effective.

In this paper we present a computational framework for generating optimized SBPs that improves on previous work [1, 2]. Specifically, we describe SBP constructions for single permutations that yield a CNF formula whose size is linear, rather than quadratic, in the number of variables in the SAT problem. We also show how that formula can be simplified by taking advantage of the sparsity of permutations. We test these improvements against earlier constructions and show that they yield much smaller SBPs and lead to significant run time reductions on many benchmark families.

The remainder of the paper is organized as follows. Section 2 presents the necessary definitions and notation. Section 3 covers previous work. The proposed efficient SBP constructions are described in Section 4. We show experimental results in Section 5, and the paper concludes in Section 6.

\section{Definitions and Notation}

Intuitively, a symmetry of a discrete object is a transformation, e.g., permutation, of its components that leaves the object intact. Symmetries are studied in abstract algebra in terms of groups [4]. A group is a set with a binary associative operation defined on it such that there is a unit element and every element has a unique inverse. In general, a set of group elements such that any other group element can be expressed as their product is called a generating set. The size of any irredundant generating set is no greater than the binary logarithm of the group size and in general is much smaller. A group $G$ 
is cyclic if it consists of an element $x$ and all elements of $G$ are powers of $x ; x$ is known as the cyclic group generator.

The symmetric group $\mathrm{S}(\Omega)$ on a finite set $\Omega$ is the group of all permutations of $\Omega$. If $\Omega=\{1, \ldots, n\}$, the group is commonly denoted by $S_{n}$. For an element of $\Omega$, its $G$-orbit is the set of elements of $\Omega$ to which it can be mapped by elements of $G$. Orbits define an equivalence relation on $\Omega$.

Permutations of $\Omega$, often denoted by lower-case Greek letters, can be written in tabular form where the elements of $\Omega$ are written in the first row and their images in the second row. For example, the image of element $i$ under the permutation $\pi$ will be denoted $i$ and written below $i$. We also use cycle notation, which can be produced from the tabular notation by (i) constructing directed edges from elements of $\Omega$ to their images, and (ii) listing the disjoint cycles of this directed graph. Single-element cycles are implicit and never listed. For example, (12)(456) can denote a permutation that swaps elements 1 and 2, and maps 4 to 5, 5 to 6 and 6 to 4 . Cycle notation is preferable to tabular notation for sparse permutations that map most elements of $\Omega$ to themselves.

A symmetry (automorphism) of a graph is a permutation of its vertices that maps edges to edges. If vertices are labeled by integers (colors), we may additionally require symmetries to preserve labels.

Consider the set of Boolean variables $x_{1}, \ldots, x_{n}$. A literal is either a variable or its negation. A clause is a disjunction of literals, e.g., $\left(x_{3}+x_{6}^{\prime}+x_{7}\right)$, and a CNF formula is a conjunction of clauses, e.g., $\left(x_{3}+x_{6}^{\prime}+x_{7}\right)\left(x_{10}+x_{11}^{\prime}\right)\left(x_{20}\right)$. A binary clause has two literals and can be viewed as an implication between variables, e.g., $\left(x_{11} \rightarrow x_{10}\right)$. The CNF-SAT decision problem seeks to find a truth assignment that satisfies a given CNF formula or to prove that the formula is unsatisfiable.

We will assume a total ordering on the variables $x_{1}, \ldots, x_{n}$ and consider the induced lexicographic ordering of the $2^{n}$ truth assignments, i.e., 0-1 strings of length $n$. We now assume that a group acts on the set of literals, subject to the Boolean consistency constraint, which requires that if $a \mapsto b$ then $a^{\prime} \mapsto b^{\prime}$ for any literals $a$ and $b$. Such an action unambiguously induces a corresponding action on the set of truth assignments. We focus on orbits of this action. The lex-leader of an orbit is defined as the lexicographically smallest element. A lex-leader predicate (LL-predicate) for the action is a Boolean function on $x_{1}, \ldots, x_{n}$ that evaluates to true only on lexleaders of orbits.

Consider a permutation on the set of literals. Given a CNF formula, we can permute literals in it, potentially changing the formula. A permutation of literals is a symmetry of a given CNF formula if Boolean consistency is observed and the formula is preserved under the permutation (in other words, every clause must map into a clause with the same polarities of literals). In particular, we consider simultaneous negations of sets of variables (phase-shifts) and compositions of permutations and phase-shifts (mixed symmetries) [1]. Given a CNF formula, we consider its group of symmetries and its corresponding action on truth assignments. A symmetry-breaking predicate (SBP) is a Boolean function that evaluates to true on at least one element from each orbit of the group of symmetries. In this work, we will consider SBPs that are expressed by CNF formulae, and the size of an SBP is taken to be the number of literals in its $\mathrm{CNF}$ formula. Observe that adding an SBP to the original CNF formula does not affect the satisfiability, but restricts the possible solutions to those selected by the SBP.
A full SBP is an SBP that selects exactly one element from each orbit; otherwise we call an SBP partial. A lex-leader SBP (LL-SBP) is an SBP that selects lex-leaders only. An LL-SBP is a full SBP. For SBPs that are not full, it is often important that they select lexleaders, among other elements. We call such SBPs partial lex-leader SBPs (PLL-SBPs).

\section{Previous Work}

\subsection{CNF Symmetries via Graph Automorphism}

Given a CNF formula, a graph is constructed such that the group of CNF symmetries is isomorphic to the group of graph automorphisms. A simple construction [1] represents every clause by a vertex of color 2, and every variable by two vertices of color 1 (one for the positive and one for the negative literal) connected by Boolean consistency edges. Every literal in the CNF formula is then represented by a bi-partite edge. The construction in [2] treats binary clauses differently. It leaves out their clausal vertices and connects their literal vertices by double-edges. Since some graph automorphism programs (e.g. GAP/NAUTY - http://www.gap-system.org/) do not allow double-edges, the work in [1] uses a model with single edges which can result in spurious graph automorphisms (one-sided error) if the original CNF formula contains binary clauses forming circular chains of implications. Fortunately, this rarely happens in $\mathrm{CNF}$ applications and spurious graph symmetries can be easily tested for [1]; in our experiments, we did not find any.

Note that the graph automorphism problem is believed to be outside $\mathrm{P}$, yet not NP-complete. In general, finding CNF symmetries is often easier than solving SAT. Furthermore, excellent graph automorphism software is currently available [6] and typically returns a small set of irredundant generators, rather than the complete set of permutations, which ensures exponential compression.

\subsection{The Lex-Leader Formulae}

The entire construction of symmetry-breaking predicates rests on the notion of lex-leader formulae introduced by Crawford et al. [2]. Given a group of symmetries $\Pi=\left\{\pi_{1}, \ldots, \pi_{m}\right\}$ for a CNF formula defined over a set of totally-ordered variables $x_{1}<x_{2}<\ldots<x_{n}$, the LL-SBP is defined as follows:

$$
\begin{aligned}
& \operatorname{PP}(\pi)=\bigwedge_{1 \leq i \leq n}\left[\bigwedge_{1 \leq j \leq i-1}\left(x_{j}=x_{j}^{\pi}\right)\right] \rightarrow\left(x_{i} \leq x_{i}^{\pi}\right) \\
& \operatorname{LL}-\operatorname{SBP}(\Pi)=\bigwedge_{1 \leq i \leq m} \operatorname{PP}\left(\pi_{i}\right)
\end{aligned}
$$

In the sequel we will refer to $\mathrm{PP}(\pi)$ as the permutation predicate for permutation $\pi$. By introducing auxiliary variables $e_{j}=\left(x_{j}=x_{j}^{\pi}\right)$, each PP can be translated to a CNF formula with $5 n$ clauses and $0.5 n^{2}+13.5 n$ literals.

In general, the CNF representations of the PPs defined in (1) tend to have duplicate clauses and even tautologies. In addition, the PPs of different permutations may have identical clauses leading to duplication in the LL-SBP defined in (2). Recognizing the existence of such redundancies, Aloul el al. [1] introduced a more efficient version of (1) that eliminates duplicate clauses and tautologies. They also noted that the LL-SBP in (2) can be replaced by a much smaller partial LL-SBP that uses only an irredundant set of generators $G(\Pi)$ for the group $\Pi$, i.e.,

$$
\operatorname{PLL}-\operatorname{SBP}(G(\Pi))=\bigwedge_{\pi_{i} \in G(\Pi)} \operatorname{PP}\left(\pi_{i}\right)
$$




\section{Improved SBP Constructions}

In this section we present several improvements to the constructions in $[1,2]$.

\subsection{Linear-Sized Permutation Predicates}

The first improvement we present is a CNF formula for PPs whose size is linear, rather than quadratic, in the number of variables. To facilitate the following derivation let $l_{i}=\left(x_{i} \leq x_{i}^{\pi}\right)$, $g_{i}=\left(x_{i} \geq x_{i}^{\pi}\right)$, and $g_{0} \equiv 1$. Noting that $e_{i}=l_{i} g_{i}$, the PP in (1) can now be expressed as:

$$
\left(g_{0} \rightarrow l_{1}\right)\left(g_{0} l_{1} g_{1} \rightarrow l_{2}\right) \ldots\left(g_{0} l_{1} \ldots l_{n-1} g_{n-1} \rightarrow l_{n}\right)
$$

Factoring out the common prefix $g_{0}$ and simplifying yields:

$$
g_{0} \rightarrow\left[l_{1} \cdot\left(g_{1} \rightarrow l_{2}\right) \ldots\left(g_{1} l_{2} \ldots l_{n-1} g_{n-1} \rightarrow l_{n}\right)\right]
$$

The recursive structure of the formula is now revealed by comparing (4) and (5). Let $p_{1}, \ldots, p_{n}$ be a sequence of bit predicates defined by:

$$
p_{i}=g_{i-1} \rightarrow l_{i} p_{i+1} \quad i=1, \ldots, n ; \quad p_{n+1}=1
$$

Note that predicate $p_{1}$ represents the entire formula (4). The satisfiability of (4) can, thus, be determined by checking the satisfiability of the following equivalent, but simpler formula:

$$
\left(p_{1}\right)\left(p_{1}=g_{0} \rightarrow l_{1} p_{2}\right) \ldots\left(p_{n}=g_{n-1} \rightarrow l_{n} p_{n+1}\right)
$$

One final simplification replaces the equalities in (7) with implications since we are only interested in satisfying each of the predicates. We thus obtain:

$$
\left(p_{1}\right)\left(p_{1} \rightarrow g_{0} \rightarrow l_{1} p_{2}\right) \ldots\left(p_{n} \rightarrow g_{n-1} \rightarrow l_{n} p_{n+1}\right)
$$

The CNF representation of (8) consists of $2 n$ 3-literal and $2 n$ 4-literal clauses for a total size of $14 n$ literals. Schematically, (8) can be viewed as a multi-level logic circuit (see Figure 1). Hence, the truth assignments that satisfy (1) are those assignments that set the circuit output $p_{1}$ to 1 . It is also interesting to note that bit predicates form a chain that is reminiscent of carry chains in ripple adders.

\subsection{Elimination of Tautologies in PPs}

For a given permutation, choosing an SBP with fewer literals is also important for empirical success. Since the permutations are typically sparse, the size of the CNF representation of (8) can be further reduced by eliminating all tautologies. We illustrate our approach with an example. Consider the permutation:

$$
\pi=\left(y_{1} y_{4}\right)\left(y_{5} y_{6}\right)\left(y_{7} y_{8} y_{9}\right)
$$

expressed in cycle notation. The permutation can be expressed in tabular form as follows:

$$
\left(\begin{array}{l}
y_{1} y_{2} y_{3} y_{4} y_{5} y_{6} y_{7} y_{8} y_{9} \\
y_{4} y_{2} y_{3} y_{1} y_{6} y_{5} y_{8} y_{9} y_{7}
\end{array}\right)
$$

We proceed to create a sequence of bit predicates $p_{1}, p_{2}, p_{3}, \ldots$ using (7). The only difference is that we eliminate the predicates of the variables that do not move. For such variables note that $l_{i}=g_{i}=1$. In this example, $l_{2}=g_{2}=l_{3}=g_{3}=1$. This immediately simplifies the predicates of those variables to:

$$
\begin{aligned}
& p_{2}=g_{1} \rightarrow l_{2} p_{3}=g_{1} \rightarrow p_{3} \\
& p_{3}=g_{2} \rightarrow l_{3} p_{4}=p_{4}
\end{aligned}
$$

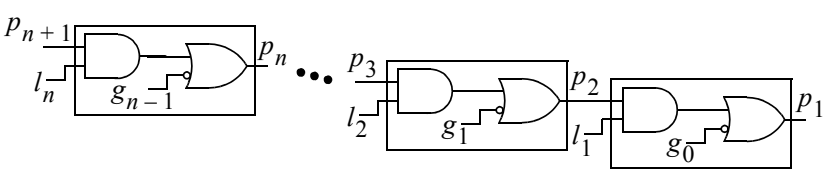

Figure 1. Circuit representation of (8).

$$
p_{1}=g_{0} \rightarrow l_{1}\left(g_{1} \rightarrow p_{4}\right)
$$

and $p_{4}$ reduces to:

$$
p_{4}=l_{4} p_{5}
$$

The pattern should now be clear. Given a permutation, where variables $y_{i}$ and $y_{j}$ move and $y_{i+1}, \ldots, y_{j-1}$ do not move, the PP can be simplified to:

$$
\begin{aligned}
& p_{i}=g_{i-1} \rightarrow l_{i}\left(g_{i} \rightarrow p_{j}\right) \\
& p_{j}=l_{j} p_{j+1}
\end{aligned}
$$

All other bit predicates, representing variables that move, use the format described in (6).

\subsection{Options for Partial Symmetry Breaking}

Breaking all symmetries may not speed up search because there are often exponentially many of them and their PLL-SBPs may be redundant [2]. Breaking enough symmetries, whose SBPs are short CNF clauses, may provide a better trade-off. Irredundant generators are good candidates for symmetries to be broken because they cannot be expressed in terms of each other, which minimizes redundancies. Alternatively, we can use the powers or compositions of generators as candidates for symmetry breaking.

Additionally, we can reduce the size of SBPs by considering only the first $k$ bits from each permutation. This is achieved by generating the SBPs up to the $k$-th predicate and setting the last used predicate to 1 .

\section{Experimental Results}

In this section, we empirically show the advantage of using the proposed SBP constructions. The experiments were performed on an AMD Athlon $1.2 \mathrm{GHz}$ machine with $1 \mathrm{~GB}$ of RAM running Linux. The run time limit for all experiments was set to 1000 seconds. The benchmarks included pigeon-hole [3], randomized Urquhart (URQ) [10], global routing (s3) [1], FPGA routing (fpga, chan) [8], and xor-chains [9]. We used the best available backtrack SAT solver Chaff [7]. Since Chaff is randomized, its run time varies, and we averaged all results over 200 independent runs.

Table 1 lists symmetry detection run times, number of symmetries, symmetry generators, and phase shifts. We use the reduction to graph automorphism from [1] which detects a wider range of symmetries than that from [2]. The table clearly shows the significant savings obtained when generators are used to represent the complete set of symmetries. Note that all generators consisted of cycles of size 2 only. The table also shows the percentage of bits that map to themselves (shown as "\%RB"). Clearly, more than $80 \%$ of the bits in all instances are redundant and can lead to significant savings in run time and memory if removed from the SBPs.

Table 1 also compares SAT-solving run times for the original CNF instance and the instances augmented with generator SBPs using the construction in equation (3), reference [1], and the proposed constructions in Sec. 4.1 and 4.2. Clearly, the addition of SBPs sig-

which allows us to express $p_{1}$ directly in terms of $p_{4}$ : 
Table 1. Search run times of CNF-SAT instances with and without PLL-SBPs (for generators only). Symmetry statistics including symmetry detection run time, number of symmetries, generators, and phase shifts are also provided.

\begin{tabular}{|c|c|c|c|c|c|c|c|}
\hline \multirow{2}{*}{ Instance } & \multirow{2}{*}{$\mathbf{V}$} & \multirow{2}{*}{$\mathrm{C}$} & \multicolumn{5}{|c|}{ Symmetry statistics } \\
\hline & & & Find (sec) & \#Sym & \#Gen & \#P.S. & $\% R B$ \\
\hline hole11 & 132 & $\overline{738}$ & 0.09 & $1.9 \mathrm{E}+16$ & 21 & 0 & $\overline{83}$ \\
\hline hole12 & 156 & 949 & 0.12 & $2.9 \mathrm{E}+18$ & 23 & 0 & 84 \\
\hline Urq3_4 & 36 & 220 & 0.10 & $5.2 \mathrm{E}+05$ & 19 & 19 & 97 \\
\hline Urq3_9 & 37 & 236 & 0.04 & $1.1 \mathrm{E}+06$ & 20 & 20 & 97 \\
\hline s3-3-3-3 & 960 & 9156 & 8.92 & $6.9 \mathrm{E}+10$ & 29 & 0 & 95 \\
\hline s3-3-3-8 & 912 & 8356 & 6.95 & $3.5 \mathrm{E}+10$ & 28 & 0 & 95 \\
\hline fpga13_10 & 195 & 905 & 0.25 & $1.9 \mathrm{E}+17$ & 28 & 0 & 88 \\
\hline fpga13_12 & 234 & 1242 & 0.81 & $9.0 \mathrm{E}+20$ & 32 & 0 & 89 \\
\hline chnl11_13 & 286 & 1742 & 0.96 & $1.2 \mathrm{E}+35$ & 45 & 0 & 90 \\
\hline chnl11_20 & 440 & 4220 & 4.48 & $1.9 \mathrm{E}+52$ & 59 & 0 & 92 \\
\hline xor1_32 & 94 & 250 & 0.17 & $4.3 \mathrm{E}+09$ & 32 & 32 & 98 \\
\hline xor1_36 & 106 & 282 & 0.34 & $6.9 \mathrm{E}+10$ & 36 & 36 & 99 \\
\hline Total & 3588 & 28296 & $\overline{23}$ & $1.9 \mathrm{E}+52$ & 372 & 107 & - \\
\hline
\end{tabular}

\begin{tabular}{|c|ccc|cc|}
\hline \multicolumn{5}{|c|}{ Chaff run time (sec) } \\
Orig & $\mathbf{( 3 )}$ & {$[\mathbf{1}]$} & Sec. 4.1 & Sec. 4.2 \\
\hline 219 & 5.43 & 0.03 & 3.52 & 0.02 \\
1000 & 11.0 & 0.04 & 8.65 & 0.02 \\
\hline 0.85 & 0.16 & 0.01 & 0.08 & 0.01 \\
12.8 & 0.05 & 0.01 & 0.02 & 0.01 \\
\hline 44.4 & 200 & 2.85 & 65.3 & 0.50 \\
21.3 & 141 & 2.61 & 138 & 0.93 \\
\hline 1000 & 517 & 0.09 & 7.70 & 0.03 \\
1000 & 1000 & 0.08 & 25.8 & 0.05 \\
\hline 788 & 882 & 0.16 & 108 & 0.05 \\
1000 & 1000 & 0.30 & 92.6 & 0.09 \\
\hline 830 & 12.6 & 1.7 & 1.73 & 1.7 \\
938 & 0.61 & 0.01 & 0.1 & 0.01 \\
\hline $\mathbf{6 8 5 4}$ & $\mathbf{3 7 7 0}$ & $\mathbf{7 . 9}$ & $\mathbf{4 5 2}$ & $\mathbf{3 . 4}$ \\
\hline
\end{tabular}

\begin{tabular}{|c|c|}
\hline \multicolumn{2}{|c|}{$\begin{array}{c}\text { Sec. 4.2 speedup over } \\
\text { Orig }\end{array}$} \\
\hline $11 \mathrm{~K}$ & 1.5 \\
$>50 \mathrm{~K}$ & 2 \\
\hline 85 & 1 \\
1280 & 1 \\
\hline 89 & 5.7 \\
23 & 2.8 \\
\hline$>33 \mathrm{~K}$ & 3 \\
$>20 \mathrm{~K}$ & 1.6 \\
\hline $16 \mathrm{~K}$ & 3.2 \\
$>11 \mathrm{~K}$ & 3.3 \\
\hline 488 & 1 \\
$94 \mathrm{~K}$ & 1 \\
\hline- & - \\
\hline
\end{tabular}

Table 2. Total size of generator-only SBPs using various SBP constructions for the instances presented in Table 1.

\begin{tabular}{|l|c|c|c|c|}
\hline$\#$ & $\mathbf{( 3 )}$ & {$[1]$} & Sec. 4.1 & Sec. 4.2 \\
\hline Var & $120 \mathrm{~K}$ & $8.6 \mathrm{~K}$ & $120 \mathrm{~K}$ & $\mathbf{8 . 6 K}$ \\
Cl & $599 \mathrm{~K}$ & $44 \mathrm{~K}$ & $478 \mathrm{~K}$ & $\mathbf{3 4 K}$ \\
Lit & $36 \mathrm{M}$ & $404 \mathrm{~K}$ & $1.7 \mathrm{M}$ & $\mathbf{1 1 9 K}$ \\
\hline
\end{tabular}

nificantly reduces the search run time, and the approach in Sec. 4.2 leads to the greatest savings in run time.

Table 2 compares the size of SBP predicates produced by the above four constructions. The construction proposed in Sec. 4.2 entails the smallest number of variables, clauses, and literals for all analyzed instances.

In order to further reduce the size of SBPs, we tested all presented instances after considering only the first $k$ bits from each permutation. Figure 2 plots the total search run time for all instances as a function of $k$. The construction proposed in Sec. 4.2 was used in the experiment. Interestingly, the total search run time decreases as additional bits are considered. Nevertheless, the performance improvements fade after considering 10 bits per permutation for almost all instances. This confirms the intuition that breaking all symmetries does not necessarily speed up the search process. In fact, breaking the symmetries for only a subset of permutations, i.e. irredundant sets of generators, and considering a limited number of bits from each permutation seems to be sufficient to significantly reduce the search run times in all cases.

Our final experiment compares the search run time and memory requirements of different choices of permutation sets to break. We considered three such sets: generators, powers of generators, and compositions of generators. Since all generators produced by the graph automorphism program were expressed as products of length2 cycles, the set of their powers did not yield additional permutations. However, the set of permutations obtained by composing the 372 generators yielded a total of 5402 permutations. Using the construction proposed in Sec. 4.2, the SBPs for these permutations consisted of $326 \mathrm{~K}$ variables, $1.3 \mathrm{M}$ clauses, and $4.5 \mathrm{M}$ literals. This significant increase in the size of the SBP was reflected in a much larger search run time: 29 seconds as opposed to 3.4 seconds for the generator-only SBP. This provides further empirical evidence for the use of generator-only SBPs to speed up satisfiability search.

\section{Conclusions}

In this work we extended and improved the framework of symmetry-breaking predicates for solving Boolean Satisfiability by con-

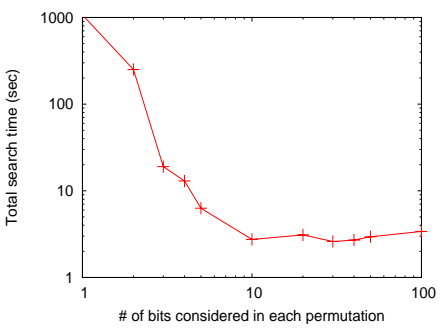

Figure 2. Total search run times of instances shown in Table 1 when only $k$ bits are considered from each generator.

structing more efficient CNF representations of symmetry-breaking predicates. The proposed techniques lead to empirical speed-ups in backtrack search and smaller memory requirements for the best available SAT solvers. Additionally, we gave new justifications of partial symmetry-breaking by generators.

\section{Acknowledgments}

This work was funded by the DARPA/MARCO Gigascale Silicon Research Center and an Agere Systems/SRC Research fellowship.

\section{References}

[1] F. Aloul, A. Ramani, I. Markov, and K. Sakallah, "Solving Difficult SAT Instances in the Presence of Symmetries," in Proc. of the Design Automation Conference (DAC), 731-736, 2002.

[2] J. Crawford, M. Ginsberg, E. Luks, and A. Roy, "Symmetry-breaking predicates for search problems," in Proc. of the Intl. Conference Principles of Knowledge Representation and Reasoning, 148-159, 1996.

[3] DIMACS Challenge benchmarks in ftp://Dimacs.rutgers.EDU/pub/ challenge/sat/benchmarks/cnf.

[4] I. Gessel and R. Stanley, "Algebraic Enumeration," in R. Graham, M. Grötschel and L. Lovász, eds, Handbook of Combinatorics, vol. 2, MIT Press, 1021-1061, 1995.

[5] E. Goldberg and Y. Novikov, "BerkMin: A fast and robust SATsolver," in Proc. of DATE, 142-149, 2002.

[6] B. McKay, "Practical Graph Isomorphism," in Congressus Numerantium, vol. 30, 45-87, 1981.

[7] M. Moskewicz, C. Madigan, Y. Zhao, L. Zhang, and S. Malik, "Chaff: Engineering an Efficient SAT Solver," in DAC, 530-535, 2001.

[8] G. Nam, F. Aloul, K. Sakallah, and R. Rutenbar, "A Comparative Study of Two Boolean Formulations of FPGA Detailed Routing Constraints," in Proc. of Intl. Symp. on Physical Design, 222-227, 2001.

[9] SAT 2002 Competition, http://www.satlive.org/SATCompetition/submittedbenchs.html

[10] A. Urquhart, "Hard Examples for Resolution," in Journal of the ACM, 34(1), 209-219, 1987. 